\title{
Contralateral progression after unilateral evacuation of bilateral chronic subdural hematomas: the volume relation ratio as prognostic factor?
}

\author{
Florian Scheichel, MD, , ${ }^{1,2}$ Branko Popadic, MD, 1,2 Karl Ungersboeck, MD, 1,2 and Franz Marhold, MD1,2 \\ 'Department of Neurosurgery, University Hospital of St. Poelten; and ${ }^{2}$ Karl Landsteiner University of Health Sciences, Krems an \\ der Donau, Lower Austria, Austria
}

\begin{abstract}
OBJECTIVE Unilateral evacuation of bilateral chronic subdural hematomas (bcSDHs) is associated with higher retreatment rates than an initial bilateral intervention. One reason for that is a possible progression in the size of the contralateral side after unilateral treatment. Thus, the authors focused their study on finding predictors of the need for contralateral retreatment.
\end{abstract}

METHODS All patients who had undergone unilateral or bilateral evacuation of bcSDHs in the Department of Neurosurgery at the University Hospital of St. Poelten during a 5-year period (7/2012 to 6/2017) were retrospectively identified. The preoperative hematoma volume was calculated using the XYZ/2 method.

RESULTS Of a total of 103 patients with bcSDHs, 61 patients underwent bilateral evacuation and 42 patients underwent unilateral evacuation. The retreatment rate after bilateral evacuation was significantly lower than that after unilateral evacuation $(14.8 \%$ vs $31 \%$, respectively; $p=0.049)$. Contralateral retreatment after unilateral evacuation was necessary in 9 patients $(21.4 \%)$. The preoperative contralateral hematoma volume was significantly higher in those patients who needed contralateral retreatment after initial unilateral evacuation $\left(68.4 \mathrm{~cm}^{3} \mathrm{vs} 27.4 \mathrm{~cm}^{3}\right.$, respectively; $\left.p<0.001\right)$. Furthermore, the so-called volume relation ratio created by dividing the smaller by the larger hematoma volume was significantly higher when contralateral retreatment became necessary ( 0.56 vs 0.21 , respectively; $p<0.001)$.

CONCLUSIONS Patients needing evacuation of bcSDHs should be considered for primary bilateral evacuation if the hematoma volume on the smaller side is greater than $40 \mathrm{~cm}^{3}$ and the subsequent volume relation ratio is greater than 0.4 .

https://thejns.org/doi/abs/10.3171/2018.6.JNS18467

KEYWORDS chronic subdural hematoma; bilateral; retreatment; trauma

$\mathrm{C}$ HRONIC subdural hematoma (cSDH) is a common diagnosis in neurosurgery. The incidence is up to 13.1 per 100,000/year with an increase in the elderly. $4,5,8$ A cSDH is a slowly progressive collection of liquefied SDH arising in $50 \%$ to $80 \%$ after minor head trauma. ${ }^{4,14,18}$ The time interval from trauma to onset of symptoms is often delayed. Known additional risk factors are antiplatelet or anticoagulant therapy, age, and brain atrophy. ${ }^{4,14}$

Bilateral cSDHs (bcSDHs) account for $17.4 \%-34.9 \%$ of cSDH cases. $7,17,19,21$

Although there is no standardized treatment for cSDH and bcSDH, indication for surgery is generally accepted in symptomatic patients. Various surgical techniques have been used for years. Burr hole craniostomy with or without insertion of a closed drainage system is the most frequent performed procedure. ${ }^{9,20}$

However, recurrence of cSDH requiring ipsilateral reoperation is common. The reported rate ranges from $9.2 \%$ to $26.5 \%,{ }^{1,2,10-12,15}$ Moreover, in bcSDHs the retreatment rate reaches almost $30 \%{ }^{7,13,19}$ Noted retreatment rates for bcSDHs in the literature depict both surgery for ipsilateral recurrence and surgery for contralateral progression after initial unilateral hematoma evacuation. Because of

ABBREVIATIONS bcSDH = bilateral chronic subdural hematoma; $C C T=$ cranial $C T ; C S D H=$ chronic subdural hematoma; $I C P=$ intracranial pressure; VR-ratio = volume relation ratio.

SUBMITTED February 18, 2018. ACCEPTED June 12, 2018.

INCLUDE WHEN CITING Published online November 23, 2018; DOI: 10.3171/2018.6.JNS18467. 
their different underlying pathomechanisms, ipsilateral recurrence and contralateral progression should be distinguished.

Recently, unilateral evacuation of bcSDHs has been identified as an independent risk factor for retreatment. ${ }^{3}$ For all patients requiring retreatment after unilateral evacuation of a bcSDH, more than $50 \%$ need contralateral hematoma evacuation. ${ }^{3}$ Thus, it seems crucial to identify those patients who could profit from initial bilateral evacuation.

To the best of our knowledge, there are no distinct parameters predicting a contralateral progression after unilateral evacuation of bcSDHs. The decision whether to evacuate one or both sides initially is mainly based on the size of each hematoma, mass effect demonstrated on imaging studies, and lateralization of symptoms. There are no standardized parameters that could be used repeatedly in different patients.

Therefore, we focused in this analysis on finding predictors for contralateral retreatment in bcSDHs after unilateral evacuation.

\section{Methods}

We performed a retrospective single-center analysis of all surgically treated patients with bcSDHs from July 1, 2012, to June 30, 2017, at the University Hospital of St. Poelten, Austria (Karl Landsteiner Private University of Health Sciences). This study was approved by the ethics commission of Lower Austria, Austria. A database query searching for specific treatment codes (craniostomy, craniotomy) was performed to identify those patients treated for cSDH, either cSDH or bcSDHs, during this 5-year period consecutively. To identify all patients with bcSDHs, the latest cranial CT (CCT) scan obtained prior to surgery was reviewed, and only patients with bcSDHs were included in the study.

The exclusion criteria were patient age younger than 20 years, a history of ventricular shunt implantation, and decompressive craniectomy or any other previous craniotomy due to a possibility of a difference in pathophysiology. In patients harboring a possible acute $\mathrm{SDH}$, all the data were meticulously reviewed. In cases of a $\mathrm{cSDH}$, which appeared dubious, the patients were excluded.

The mean radiological follow-up duration was 172.8 days.

\section{Preoperative Imaging}

All patients underwent CCT before surgery. We analyzed the cSDH in terms of acuity, size, volume, location density, and midline shift on the last CCT scan before surgery.

The hematoma density of each side was classified as homogeneous, separated, laminar, or membranous based on the description of Nakaguchi et al. ${ }^{10}$ The preoperative hematoma volumes were calculated on the last CT scan acquired before surgery using the XYZ/2 method, which has been described to be valid for cSDH by Sucu et al. ${ }^{16}$ To calculate the hematoma volume, we identified the depth by multiplying the number of axial slices on which the hematoma was visible with the slice thickness.
The depth was multiplied by the maximum length on any slice and the maximum width on any slice and then divided by 2 .

\section{Volume Relation Ratio}

To determine if there was interaction between the individual volume and mass effect of both hematomas, we defined a variable called the volume relation ratio (VR-ratio). The VR-ratio was calculated by dividing the volume of the smaller contralateral hematoma by the volume of the larger ipsilateral one. To the best of our knowledge, this was done for the first time in cSDHs.

\section{Patient Characteristics and Surgical Treatment}

Demographic and clinical data including sex, age, symptoms, anticoagulant or antiplatelet therapy, history of head trauma, and outcome were culled from medical records. Surgical data included side of evacuation, surgical procedure, and usage of a closed drainage system. Indication for surgery and the decision of whether to perform burr hole craniostomies or craniotomies with or without insertion of a drainage system were made by the attending neurosurgeon.

The standard surgical procedure for cSDH at our institution consists of single- or double-burr hole craniostomy and insertion of a closed drainage system. In cases involving rather small hematoma volumes and intraoperative reexpansion of brain parenchyma after hematoma evacuation, the attending surgeon could decide against a drainage system when it could not be safely inserted. The attending surgeon may have decided to perform a craniotomy in a case of distinct membranous cSDH.

\section{Retreatment}

Retreatment was defined as every subsequent surgery necessary due to a progressive contralateral hematoma, recurrence of the evacuated ipsilateral hematoma, or incomplete evacuation of the ipsilateral hematoma with remaining mass effect. Information regarding retreatment included side of retreatment (ipsi- or contralateral), time to retreatment, and preoperative hematoma volume on the latest CT scan before the second surgery for contralateral retreatment. Follow-up CT scans and outpatient reports were analyzed to find patients with recurrence.

Patients who had undergone unilateral hematoma evacuation of bcSDHs were then divided into two groups: group A included patients with contralateral retreatment and group B included patients without contralateral retreatment. Attributes were compared between the groups to find predictors for contralateral retreatment after unilateral evacuation.

\section{Statistical Analysis}

For statistical analysis, we used SPSS statistical software (IBM Corp.). For qualitative variables, a chi-square test was performed; for continuous variables, a Student ttest was executed. A p value $<0.05$ was considered statistically significant. Cutoff values were found with receiver operating characteristic curve analysis. 
TABLE 1. Clinical, demographic, and surgical data

\begin{tabular}{|c|c|c|}
\hline Variable & $\begin{array}{l}\text { No. of Cases or } \\
\text { Mean Value }\end{array}$ & $\begin{array}{l}\% \text { or } \\
\text { SD }\end{array}$ \\
\hline Patients & 103 & 100 \\
\hline \multicolumn{3}{|l|}{ Sex } \\
\hline Male & 69 & 67.0 \\
\hline Female & 34 & 33.0 \\
\hline Mean age in yrs & 76.8 & \pm 9.4 \\
\hline \multicolumn{3}{|l|}{ Clinical presentation } \\
\hline Headache & 35 & 34.0 \\
\hline Nausea \& vomiting & 7 & 6.8 \\
\hline Hemiparesis & 33 & 32.0 \\
\hline Neuropsychological changes & 66 & 64.1 \\
\hline Clouding of consciousness & 22 & 21.4 \\
\hline \multicolumn{3}{|l|}{ CT density, right side } \\
\hline Homogeneous & 43 & 41.7 \\
\hline Laminar & 15 & 14.6 \\
\hline Separated & 29 & 28.2 \\
\hline Membranous & 16 & 15.5 \\
\hline \multicolumn{3}{|l|}{ CT density, left side } \\
\hline Homogeneous & 38 & 36.9 \\
\hline Laminar & 22 & 21.4 \\
\hline Separated & 23 & 22.3 \\
\hline Membranous & 20 & 19.4 \\
\hline \multicolumn{3}{|l|}{ Surgery location } \\
\hline Unilateral evacuation & 42 & 40.8 \\
\hline Right & 17 & \\
\hline Left & 25 & \\
\hline Bilateral evacuation & 61 & 59.2 \\
\hline \multicolumn{3}{|l|}{ Mean preop hematoma vol, $\mathrm{cm}^{3}$} \\
\hline \multicolumn{3}{|l|}{ Bilateral evacuation } \\
\hline Right hematoma vol & 120.8 & \pm 47.1 \\
\hline Left hematoma vol & 123.3 & \pm 49.7 \\
\hline \multicolumn{3}{|l|}{ Right evacuation } \\
\hline Right hematoma vol & 136.6 & \pm 40.3 \\
\hline Left hematoma vol & 33.7 & \pm 23.4 \\
\hline \multicolumn{3}{|l|}{ Left evacuation } \\
\hline Right hematoma vol & 38.7 & \pm 25.8 \\
\hline Left hematoma vol & 136.3 & \pm 49.7 \\
\hline \multicolumn{3}{|l|}{ Surgical procedure in bilateral op } \\
\hline 1-burr hole craniostomy & 39 & 63.9 \\
\hline 2-burr hole craniostomy & 15 & 24.6 \\
\hline Craniotomy & 7 & 11.5 \\
\hline Drain & 58 & 95.1 \\
\hline No drain & 3 & 4.9 \\
\hline \multicolumn{3}{|l|}{ Surgical procedure in unilateral op } \\
\hline 1-burr hole craniostomy & 24 & 57.1 \\
\hline 2-burr hole craniostomy & 9 & 21.4 \\
\hline Craniotomy & 9 & 21.4 \\
\hline Drain & 39 & 92.9 \\
\hline No drain & 3 & 7.1 \\
\hline
\end{tabular}

TABLE 2. Comparison of groups $A$ and $B$

\begin{tabular}{|c|c|c|c|}
\hline \multirow[b]{2}{*}{ Variable } & \multicolumn{2}{|c|}{$\begin{array}{c}\text { No. of Patients (\%) or } \\
\text { Mean Value }\end{array}$} & \multirow[b]{2}{*}{$\begin{array}{c}p \\
\text { Value }\end{array}$} \\
\hline & $\begin{array}{c}\text { Contralat } \\
\text { Retreatment } \\
(\%)\end{array}$ & $\begin{array}{c}\text { No Contralat } \\
\text { Retreatment } \\
(\%)\end{array}$ & \\
\hline Patients & $9(21.4 \%)$ & $33(78.6 \%)$ & \\
\hline Sex & & & NS \\
\hline Male & $6(66.7 \%)$ & $20(60.6 \%)$ & \\
\hline Female & $3(33.3 \%)$ & $13(39.4 \%)$ & \\
\hline $\begin{array}{l}\text { Density of the contralat hema- } \\
\text { toma on preop CT scan }\end{array}$ & & & NS \\
\hline Homogeneous & $5(55.6 \%)$ & $14(42.4 \%)$ & \\
\hline Laminar & $0(0 \%)$ & $12(36.4 \%)$ & \\
\hline Separated & $3(33.3 \%)$ & $3(9.1 \%)$ & \\
\hline Membranous & $1(11.1 \%)$ & $4(12.1 \%)$ & \\
\hline Midline shift, mm & $7.6 \pm 5.8$ & $7.4 \pm 3.6$ & NS \\
\hline $\begin{array}{l}\text { Anticoagulant or antiplatelet } \\
\text { therapy }\end{array}$ & & & NS \\
\hline Yes & $8(88.9 \%)$ & $20(60.6 \%)$ & \\
\hline No & $1(11.1 \%)$ & $13(39.4 \%)$ & \\
\hline Side of evacuation & & & NS \\
\hline Right & $3(33.3 \%)$ & $14(42.4 \%)$ & \\
\hline Left & $6(66.7 \%)$ & $19(57.6 \%)$ & \\
\hline Surgical procedure & & & NS \\
\hline 1-burr hole craniostomy & $6(66.7 \%)$ & $18(54.5 \%)$ & \\
\hline 2-burr hole craniostomy & $0(0 \%)$ & $9(27.3 \%)$ & \\
\hline Craniotomy & $3(33.3 \%)$ & $6(18.2 \%)$ & \\
\hline Drain used & & & NS \\
\hline Yes & $8(88.9 \%)$ & $31(93.9 \%)$ & \\
\hline No & $1(11.1 \%)$ & $2(6.1 \%)$ & \\
\hline Ipsilat hematoma vol, $\mathrm{cm}^{3}$ & $121.0 \pm 31.4$ & $140.6 \pm 48.3$ & NS \\
\hline Contralat hematoma vol, $\mathrm{cm}^{3}$ & $68.4 \pm 24.2$ & $27.4 \pm 15.9$ & $<0.001$ \\
\hline VR-ratio (smaller vol/larger vol) & $0.56 \pm 0.11$ & $0.21 \pm 0.12$ & $<0.001$ \\
\hline
\end{tabular}

\section{Results}

A total of 103 patients with bcSDHs were included in this study. Of these 103 patients, 69 (67\%) were male and 34 (33\%) were female. Their age ranged from 21.7 to 92.9 years (mean 76.8 years). Preoperative antiplatelet or anticoagulant therapy was administered in 59 patients $(57.3 \%)$. Head trauma was noted in 73 patients $(70.9 \%)$.

Clinical, demographic, and surgical data are summarized in Table 1.

\section{Hematoma Volumes}

The mean hematoma volume was $120.8 \mathrm{~cm}^{3}( \pm 47.1$ $\mathrm{cm}^{3}$ [SD]) on the right side and $123.3 \mathrm{~cm}^{3}\left( \pm 49.7 \mathrm{~cm}^{3}\right)$ on the left when bilateral evacuation was performed. In patients who underwent unilateral evacuation, the mean hematoma volumes were $136.6 \mathrm{~cm}^{3}\left( \pm 40.3 \mathrm{~cm}^{3}\right)$ on the 

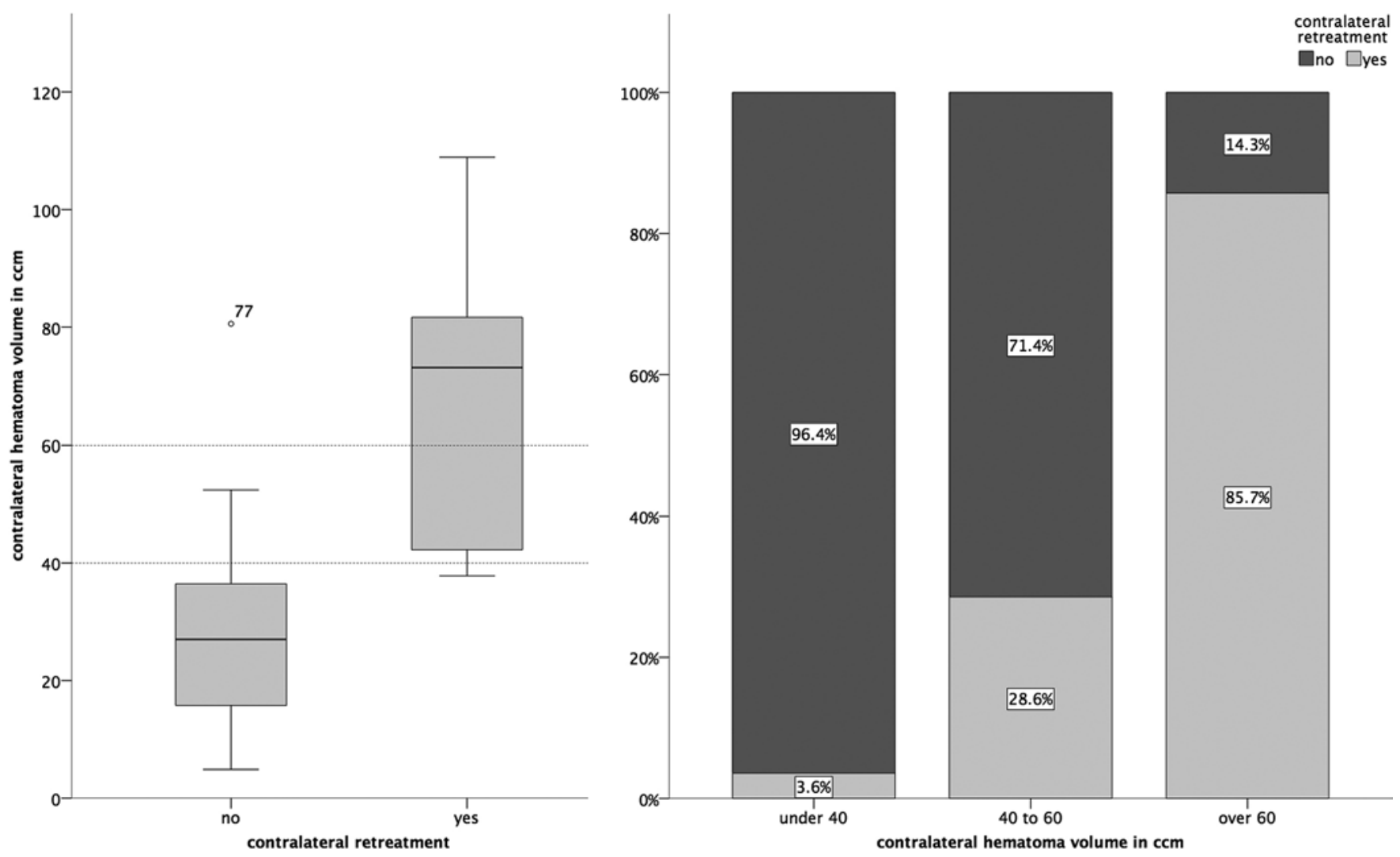

FIG. 1. Left: Box plot illustrating the preoperative contralateral hematoma volume in patients with and without contralateral retreatment after unilateral evacuation of bcSDHs. Right: Bar graph showing contralateral retreatment rates for patients with a preoperative contralateral hematoma volume of less than $40 \mathrm{~cm}^{3}$, between 40 and $60 \mathrm{~cm}^{3}$, and greater than $60 \mathrm{~cm}^{3}$. $\mathrm{ccm}=\mathrm{cubic}$ centimeters $\left(\mathrm{cm}^{3}\right)$.

right side and $33.7 \mathrm{~cm}^{3}\left( \pm 23.4 \mathrm{~cm}^{3}\right)$ on the left side after right-sided evacuation and $38.7 \mathrm{~cm}^{3}\left( \pm 25.8 \mathrm{~cm}^{3}\right)$ on the right and $136.3 \mathrm{~cm}^{3}\left( \pm 49.7 \mathrm{~cm}^{3}\right)$ on the left after left-sided evacuation.

\section{Side of Evacuation and Procedure}

Initial bilateral hematoma evacuation was performed in 61 patients $(59.2 \%)$, whereas initial unilateral evacuation was performed in 42 patients $(40.8 \%)$. Of these unilateral evacuated bcSDHs, slightly more patients underwent leftsided evacuation $(59.5 \%, 25$ cases) than right-sided evacuation $(40.5 \%, 17$ cases).

In patients who initially underwent bilateral hematoma evacuation, craniotomy on at least one side was performed in $11.5 \%$ (7 cases), a single burr hole was made in $63.9 \%$ (39 cases), and double burr holes were created in $24.6 \%$ (15 cases).

In patients who underwent unilateral evacuation, craniotomies were performed in $21.4 \%$ ( 9 cases), a single burr hole was created in $57.1 \%$ ( 24 cases), and double burr holes were made in $21.4 \%$ ( 9 cases).

A drainage system was used in $94.2 \%$ of the patients (n =97). In terms of frequency of drainage insertion, there was no significant difference between patients who underwent unilateral $(92.9 \%, 39$ cases) or bilateral $(95.1 \%, 58$ cases) evacuation.

\section{Retreatment Rates}

The overall retreatment rate was $21.4 \%$ ( 22 cases). The retreatment rate for patients who had undergone bilateral evacuation was $14.8 \%$ (9 cases), which was significantly lower than that for patients who had undergone unilateral evacuation $(31 \%, 13$ cases) $(\mathrm{p}=0.049)$. The majority of these 13 cases ( 9 cases, 69.2\%) needed retreatment on the contralateral side, and the remaining 4 cases (30.8\%) needed ipsilateral retreatment.

\section{Contralateral Retreatment After Unilateral Evacuation}

The group that needed contralateral retreatment after unilateral surgery ( $n=9$, group A) was compared with the group that did not need contralateral retreatment $(\mathrm{n}=$ 32 , group B). The results are listed in Table 2. Differences in sex, density shown on the preoperative CT scan, anticoagulation and/or antiplatelet status, surgical procedure, midline shift, and hematoma volume of the hematoma on the evacuated side did not reach statistical significance.

The mean preoperative hematoma volume on the untreated, contralateral side was significantly larger in those who needed contralateral retreatment $\left(68.4 \mathrm{~cm}^{3}\right.$ vs 27.4 $\left.\mathrm{cm}^{3} ; \mathrm{p}<0.001\right)$.

The ratio between the smaller, contralateral hematoma volume and the larger, ipsilateral evacuated hema- 
toma volume was significantly higher in group A than in group B and is therefore called VR-ratio ( 0.56 vs 0.21 ; $\mathrm{p}<0.001$ ).

A cutoff value for the VR-ratio of 0.4 produced a sensitivity of 1 and a specificity of 0.91 for contralateral retreatment (area under the receiver operating characteristic curve $0.976,95 \%$ CI $0.938-1.000 ; \mathrm{p}<0.001$ ).

The mean time to contralateral retreatment after unilateral evacuation was 28 days ( \pm 16.7 days). In one patient the hematoma volume before contralateral retreatment could not be assessed because the patient only had hard copies of the preoperative CT scans that were not accessible retrospectively. The mean hematoma volume of the progressive contralateral side of the remaining 8 patients was $148.6 \mathrm{~cm}^{3}\left( \pm 39.5 \mathrm{~cm}^{3}\right)$ on the CT scan obtained before reoperation.

\section{Discussion}

Despite the fact that $\mathrm{cSDH}$ is such a frequent condition in neurosurgery, there is no standard protocol for deciding in which patients surgical evacuation should be performed. The decision is mainly based on parameters such as the size of the hematoma, its mass effect, and midline shift on CT scans, as well as neurological symptoms and the patient's clinical condition.

In patients with bcSDHs, focal neurological deficits are less common, while symptoms due to high intracranial pressure (ICP), such as vomiting and nausea, are more frequent. ${ }^{7,17}$ Furthermore, midline shift is not as common and distinct in cases of bcSDH. $.7,7,17$

In our study the mean hematoma volumes in patients in whom bilateral surgical evacuation was performed were $120.8 \mathrm{~cm}^{3}$ (right) and $123.3 \mathrm{~cm}^{3}$ (left). Unilateral evacuation was performed in patients with mean hematoma volumes of $136.6 \mathrm{~cm}^{3}$ (right) and $136.3 \mathrm{~cm}^{3}$ (left) (Table 1). Even though there were relatively high standard deviations, this shows that the indication for surgical treatment of bcSDHs was comparable across the groups.

A recent study concerning bcSDHs that included 264 patients showed an overall retreatment rate of $21.6 \%$. Additionally, unilateral evacuation of bcSDHs was identified as an independent risk factor for surgical retreatment. The authors did not find any predictors of contralateral retreatment after unilateral evacuation. ${ }^{3}$ On the other hand, the increased risk of injuring the underlying brain tissue or missing a rather small cSDH has to be taken into account when considering initial bilateral evacuation.

In our study, the retreatment rate after unilateral evacuation of a bcSDH was $31.0 \%$ and was therefore significantly higher than after bilateral evacuation (14.8\%).

The ipsilateral reoperation rate after unilateral evacuation of a bcSDH was only $9.5 \%$ (4 cases) and thus close to the reoperation rate in unilateral cSDH shown in a recent meta-analysis $(11.5 \%))^{2}$ The contralateral retreatment rate after unilateral evacuation was $21.4 \%$ ( 9 cases). Contralateral retreatment became necessary due to a progression in size of the untreated hematoma from a mean volume of $68.4 \mathrm{~cm}^{3}\left( \pm 24.2 \mathrm{~cm}^{3}\right)$ before initial unilateral surgery to $148.6 \mathrm{~cm}^{3}\left( \pm 39.5 \mathrm{~cm}^{3}\right)$ before retreatment. The decision of whether to simultaneously evacuate the smaller hematoma
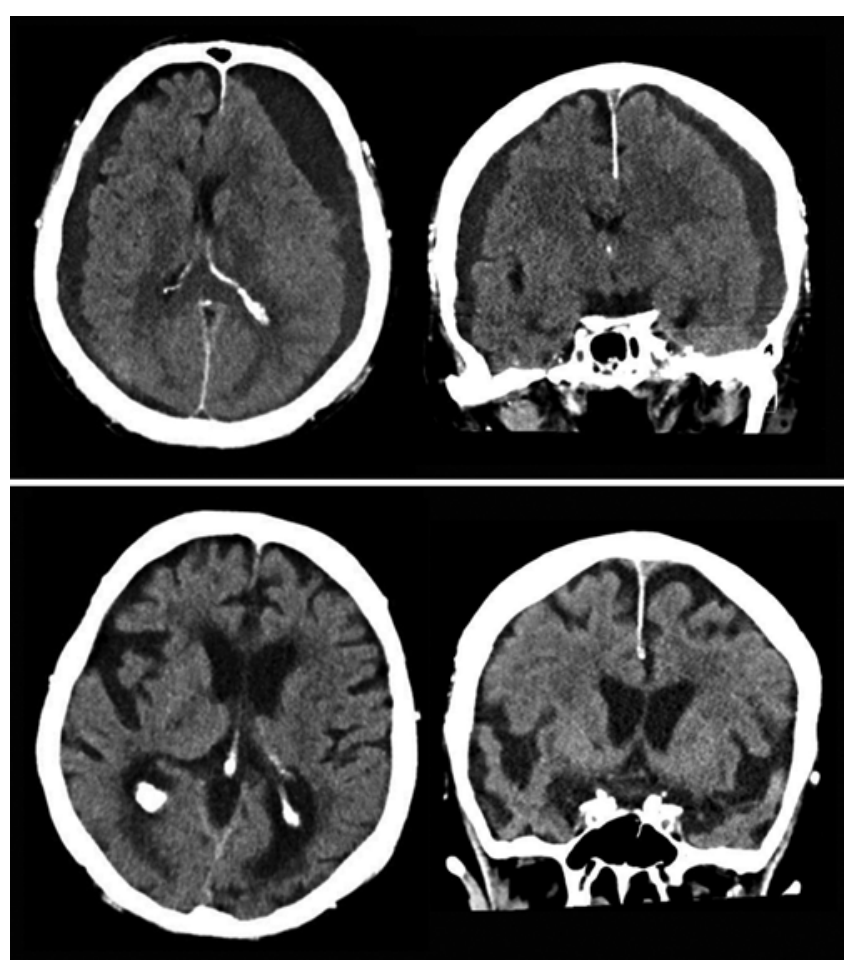

FIG. 2. Upper: CT scans obtained in a 92-year-old man with bcSDHs. Despite relatively high absolute hematoma volumes of $80.6 \mathrm{~cm}^{3}$ on the right side and $216.5 \mathrm{~cm}^{3}$ on the left side, the VR-ratio was only 0.37 . The patient underwent single-burr hole evacuation of the left-sided hematoma and placement of a closed drainage system. Lower: CT scans showing complete remission of the contralateral right-sided hematoma 14 months after the unilateral evacuation. This case illustrates the limitation of using only absolute hematoma volume numbers for decision making to evacuate the contralateral hematoma.

in bcSDH is not based on any studies, and to date there are no parameters that predict a contralateral progression after unilateral evacuation.

In our study there was a statistically significant difference in the mean contralateral preoperative hematoma volume between groups A (contralateral retreatment) and B (no contralateral retreatment) (Fig. 1 left; Table 2). The contralateral retreatment rate was only $3.6 \%$ if the contralateral hematoma volume was under $40 \mathrm{~cm}^{3}$. While it seems obvious that a larger hematoma will more likely need surgery in the future, our data indicate a cutoff at $40 \mathrm{~cm}^{3}$ and $60 \mathrm{~cm}^{3}$ (Fig. 1 right). With a contralateral hematoma volume between $40 \mathrm{~cm}^{3}$ and $60 \mathrm{~cm}^{3}$ or over 60 $\mathrm{cm}^{3}$, the retreatment rates increased to $28.6 \%$ and $85.7 \%$, respectively.

One reason for the higher retreatment rate after unilateral treatment could be a progression of the contralateral hematoma due to a reduction in ICP owing to unilateral evacuation. One could expect that the larger the evacuated ipsilateral hematoma, the higher the pressure relief on the contralateral side, and therefore the hematoma would be more likely to expand regardless of the size of the contralateral hematoma. However, there was no significant difference in ipsilateral hematoma volume between group A and group B $\left(121.0 \mathrm{~cm}^{3}\right.$ vs $140.6 \mathrm{~cm}^{3}$; Table 2). A possible explanation for this may be that the 

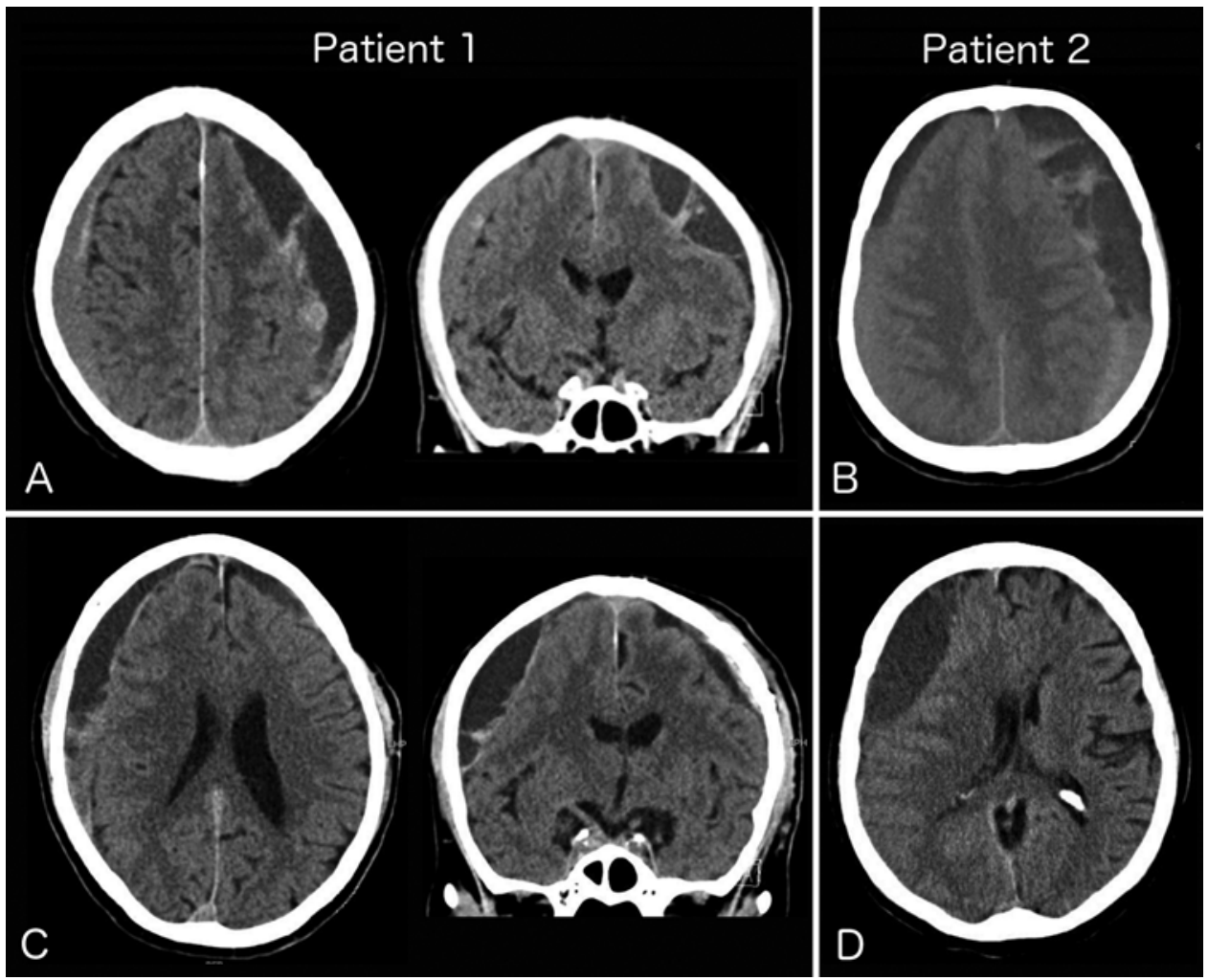

FIG. 3. CT scans obtained in two representative cases. A: CCT scan acquired in a 74-year-old man with bcSDHs. The hematoma volumes on the left and right sides were $93.4 \mathrm{~cm}^{3}$ and $64.4 \mathrm{~cm}^{3}$, respectively (VR-ratio 0.69). Due to hemiparesis on the right side, the patient was initially treated with unilateral left-sided hematoma evacuation through a craniotomy and a closed drainage system. B: CCT scan showing bcSDHs in an 82-year-old man who barely exhibited any symptoms (hematoma volumes $149.6 \mathrm{~cm}^{3}$ [left] and $73.1 \mathrm{~cm}^{3}$ [right]; VR-ratio 0.49). The patient underwent left-sided single-burr hole craniostomy and placement of a closed drainage system. C and D: Scans obtained in the same patients as on A and B, respectively, showing progression of the contralateral subdural hematoma 17 and 40 days after their initial surgery. The hematoma volumes have increased to $133.7 \mathrm{~cm}^{3}$ and $124.1 \mathrm{~cm}^{3}$, respectively, making an additional evacuation necessary.

contralateral hematoma does not always have the tendency to progress or is, at the time of unilateral surgery, already regressing. The contralateral hematoma seems to expand only when the elevated ICP is the main factor that prevented it from doing so. Thus it seems to be a complex relationship between both hematomas and the brain parenchyma that needs to be analyzed. In the presence of mass effect through a bcSDH, brain atrophy cannot easily be assessed on imaging studies. Therefore, a ratio determined by dividing the volume of the smaller hematoma by the volume of the larger hematoma was generated to describe the relation between both hematomas and was named VR-ratio by the authors. The VR-ratio works as a possible surrogate parameter for the pressure relation between both hematomas. The higher the VR-ratio the closer was the volume of the contralateral, smaller hematoma to the larger one, which was large enough to indicate the need for surgical evacuation. Furthermore, this ratio draws the attention away from the absolute hematoma volumes to the relation of each side's hematoma volume. Absolute volume numbers are more dependent on individual factors such as brain atrophy or the capacity (elasticity) of the brain to compensate additional mass effect. The possibility of compensation for a distinct hema- toma volume is different in every individual patient and shows great variations. A small hematoma can produce a distinct mass effect and cause symptoms in a young patient, while, in the presence of brain atrophy, a rather large hematoma can be compensated for easily. The VRratio removes the individual factor from the equation and produces an objective parameter to show the influence of one hematoma on the other. In combination with the absolute hematoma volumes, the VR-ratio tries to describe the interaction of all intracranial components.

In patients in whom contralateral retreatment became necessary, the VR-ratio was significantly higher (0.56 vs 0.21 ), with a cutoff value of 0.4 (a sensitivity of 1 and a specificity of 0.91 ).

Figure 2 shows CT scans obtained in a patient who underwent unilateral surgery because of rapid clinical deterioration; the preoperative contralateral hematoma volume was $80.6 \mathrm{~cm}^{3}$. Taking only the absolute numbers of the hematoma volumes into account, this patient should have initially undergone evacuation on both sides. The VR-ratio in this patient was 0.37 . In the presence of distinct brain atrophy, however, this patient did not need a contralateral reoperation and showed resorption of the hematoma with conservative treatment. 


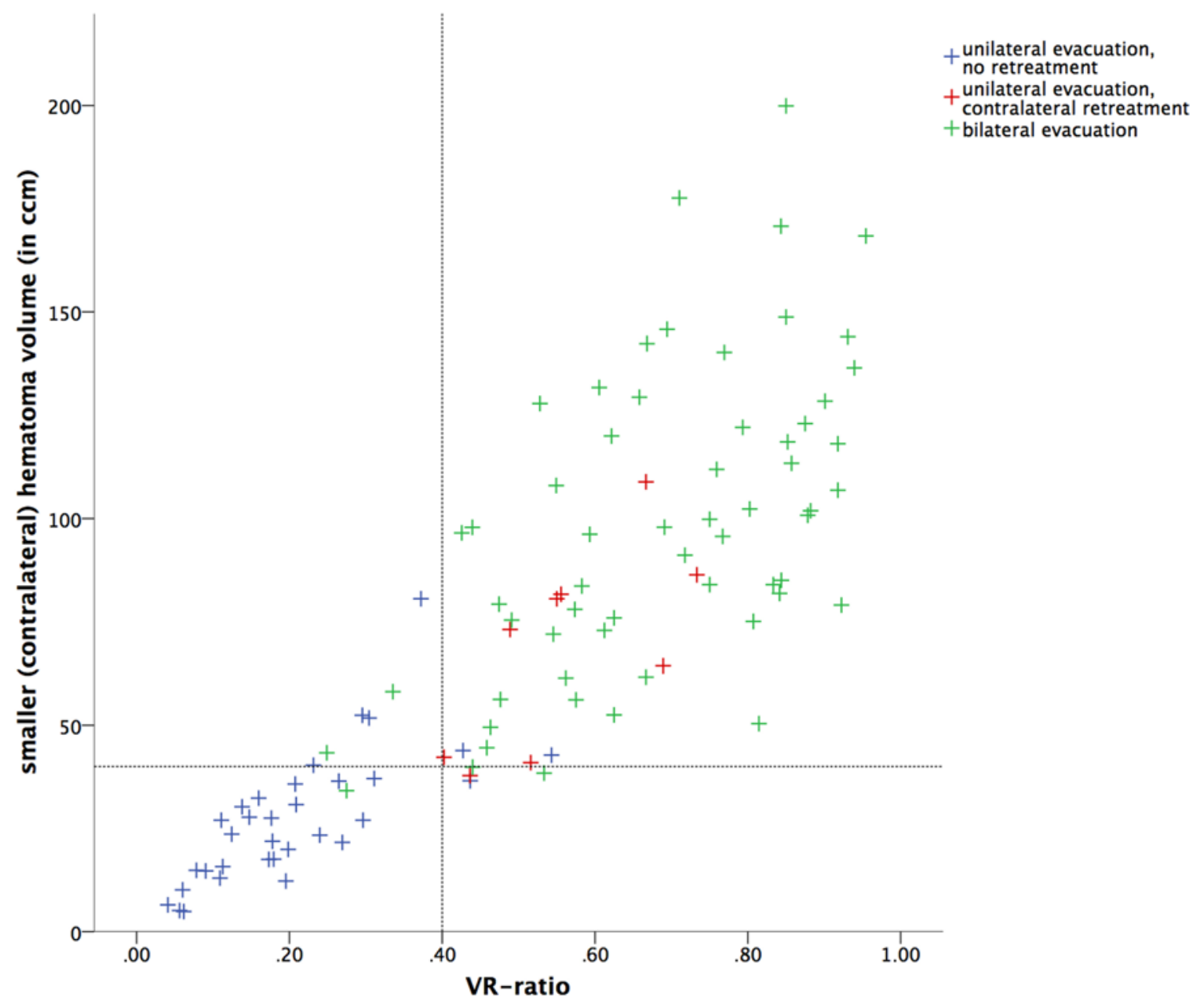

FIG. 4. Graph illustrating the preoperative hematoma volume of the smaller, contralateral hematoma and the VR-ratio for all patients. The horizontal dotted line indicates a hematoma volume of $40 \mathrm{~cm}^{3}$ and the vertical dotted line indicates a VR-ratio of 0.4 . Patients needing contralateral retreatment after an initially unilateral evacuation of their bcSDHs (group A, red) are almost entirely located in the upper right portion of the graph, meaning that these patients showed a contralateral hematoma volume exceeding $40 \mathrm{~cm}^{3}$ and a VR-ratio of at least 0.4 . Patients who underwent initially bilateral evacuation of their bcSDHs (green) are mainly located in the upper right section, meaning larger hematomas on both sides. In the bottom left portion of the graph are mostly patients who underwent unilateral evacuation and did not need contralateral retreatment (group B, blue). The upper left area is nearly empty because this would indicate a condition in which the sum of both hematoma volumes would reach fatal levels. In contrast, the right bottom portion is spared because locations there would indicate patients with hematoma volumes of less than $40 \mathrm{~cm}^{3}$ on each side. Such small hematomas would probably not cause any symptoms and therefore the patients would not have needed surgical evacuation.

In contrast to that, 3 patients who needed subsequent contralateral retreatment after initial unilateral evacuation had small contralateral hematoma volumes of 41.0 $\mathrm{cm}^{3}, 42.2 \mathrm{~cm}^{3}$, and $37.8 \mathrm{~cm}^{3}$ but VR-ratios of $0.52,0.4$, and 0.44 , respectively. The latter two patients additionally were relatively young (21.7 and 56.9 years) and had no brain atrophy; despite having small bcSDHs, they had distinct mass effect. Figure 3 shows 2 other patients initially treated with unilateral hematoma evacuation. Their VR-ratio was at least 0.4 , and both of them subsequently needed contralateral retreatment.

Figure 4 shows the correlation between the volume of the contralateral smaller hematoma and the VR-ratio in patients who underwent unilateral or bilateral evacuation. The figure illustrates how patients who needed contralat- eral retreatment after initial unilateral evacuation had a contralateral hematoma volume greater than $40 \mathrm{~cm}^{3}$ and a VR-ratio greater than 0.4 in the vast majority of cases. Such patients would probably have profited from initial bilateral evacuation.

While the VR-ratio is produced by dividing one hematoma volume by the other, the ratio tries to characterize the pressure relation of the intracranial compartments in the presence of bcSDHs. The benefit of the VR-ratio, therefore, depends on the surgical indication. In this retrospective study, this decision was made by the neurosurgeon on duty and was based on the presence of mass effect in imaging studies and clinical symptoms. The decision of whether or not bcSDHs should be treated surgically was not an issue of this study. 


\section{Limitations}

The primary limitation of this study is its retrospective design.

\section{Conclusions}

To date, the decision of whether to perform bilateral or unilateral drainage of bcSDHs is based on clinical and radiological parameters, but reproducible objective measurements are lacking. Our study provides evidence that a primary bilateral evacuation should be considered if the smaller hematoma is greater than $40 \mathrm{~cm}^{3}$ and the relation between the smaller and larger hematoma volume (VRratio) is greater than 0.4 .

\section{References}

1. Abouzari M, Rashidi A, Rezaii J, Esfandiari K, Asadollahi $\mathrm{M}$, Aleali $\mathrm{H}$, et al: The role of postoperative patient posture in the recurrence of traumatic chronic subdural hematoma after burr-hole surgery. Neurosurgery 61:794-797, 2007

2. Almenawer SA, Farrokhyar F, Hong C, Alhazzani W, Manoranjan B, Yarascavitch B, et al: Chronic subdural hematoma management: a systematic review and meta-analysis of 34,829 patients. Ann Surg 259:449-457, 2014

3. Andersen-Ranberg NC, Poulsen FR, Bergholt B, Hundsholt T, Fugleholm K: Bilateral chronic subdural hematoma: unilateral or bilateral drainage? J Neurosurg 126:1905-1911, 2017

4. Asghar M, Adhiyaman V, Greenway MW, Bhowmick BK, Bates A: Chronic subdural haematoma in the elderly-a North Wales experience. J R Soc Med 95:290-292, 2002

5. Cousseau DH, Echevarría Martín G, Gaspari M, Gonorazky SE: [Chronic and subacute subdural haematoma. An epidemiological study in a captive population.] Rev Neurol 32:821-824, 2001 (Span)

6. Hsieh CT, Su IC, Hsu SK, Huang CT, Lian FJ, Chang CJ: Chronic subdural hematoma: differences between unilateral and bilateral occurrence. J Clin Neurosci 34:252-258, 2016

7. Huang YH, Yang KY, Lee TC, Liao CC: Bilateral chronic subdural hematoma: what is the clinical significance? Int J Surg 11:544-548, 2013

8. Kudo H, Kuwamura K, Izawa I, Sawa H, Tamaki N: Chronic subdural hematoma in elderly people: present status on Awaji Island and epidemiological prospect. Neurol Med Chir (Tokyo) 32:207-209, 1992

9. Liu W, Bakker NA, Groen RJM: Chronic subdural hematoma: a systematic review and meta-analysis of surgical procedures. J Neurosurg 121:665-673, 2014

10. Nakaguchi H, Tanishima T, Yoshimasu N: Factors in the natural history of chronic subdural hematomas that influence their postoperative recurrence. J Neurosurg 95:256-262, 2001

11. Nakaguchi H, Tanishima T, Yoshimasu N: Relationship between drainage catheter location and postoperative recurrence of chronic subdural hematoma after burr-hole irrigation and closed-system drainage. J Neurosurg 93:791-795, 2000
12. Rohde V, Graf G, Hassler W: Complications of burr-hole craniostomy and closed-system drainage for chronic subdural hematomas: a retrospective analysis of 376 patients. Neurosurg Rev 25:89-94, 2002

13. Schwarz F, Loos F, Dünisch P, Sakr Y, Safatli DA, Kalff R, et al: Risk factors for reoperation after initial burr hole trephination in chronic subdural hematomas. Clin Neurol Neurosurg 138:66-71, 2015

14. Sim YW, Min KS, Lee MS, Kim YG, Kim DH: Recent changes in risk factors of chronic subdural hematoma. J Korean Neurosurg Soc 52:234-239, 2012

15. Stanišic M, Pripp AH: A reliable grading system for prediction of chronic subdural hematoma recurrence requiring reoperation after initial burr-hole surgery. Neurosurgery 81:752-760, 2017

16. Sucu HK, Gokmen M, Gelal F: The value of XYZ/2 technique compared with computer-assisted volumetric analysis to estimate the volume of chronic subdural hematoma. Stroke 36:998-1000, 2005

17. Tsai TH, Lieu AS, Hwang SL, Huang TY, Hwang YF: A comparative study of the patients with bilateral or unilateral chronic subdural hematoma: precipitating factors and postoperative outcomes. J Trauma 68:571-575, 2010

18. Tseng JH, Tseng MY, Liu AJ, Lin WH, Hu HY, Hsiao SH: Risk factors for chronic subdural hematoma after a minor head injury in the elderly: a population-based study. Biomed Res Int 2014:218846, 2014

19. Tugcu B, Tanriverdi O, Baydin S, Hergunsel B, Günaldı Ö, Ofluoglu E, et al: Can recurrence of chronic subdural hematoma be predicted? A retrospective analysis of 292 cases. J Neurol Surg A Cent Eur Neurosurg 75:37-41, 2014

20. Weigel R, Schmiedek P, Krauss JK: Outcome of contemporary surgery for chronic subdural haematoma: evidence based review. J Neurol Neurosurg Psychiatry 74:937-943, 2003

21. Zderkiewicz E, Czochra M, Koźniewska H, Turowski K: [Chronic bilateral subdural hematoma.] Neurol Neurochir Pol 14:543-546, 1980 (Polish)

\section{Disclosures}

The authors report no conflict of interest concerning the materials or methods used in this study or the findings specified in this paper.

\section{Author Contributions}

Conception and design: Scheichel. Acquisition of data: Scheichel, Popadic. Analysis and interpretation of data: Scheichel. Critically revising the article: Marhold, Scheichel. Reviewed submitted version of manuscript: Marhold, Scheichel, Ungersboeck. Approved the final version of the manuscript on behalf of all authors: Marhold. Statistical analysis: Scheichel. Study supervision: Marhold.

\section{Correspondence}

Franz Marhold: University Hospital of St. Poelten, Lower Austria, Austria. franz.marhold@stpoelten.lknoe.at. 